\title{
Neighbourhood social interactions and risk of acute myocardial infarction
}

\author{
B Chaix, ${ }^{1,2}$ M Lindström, ${ }^{1}$ M Rosvall, ${ }^{3}$ J Merlo ${ }^{1}$
}

${ }^{1}$ Community Medicine and Public Health, Department of Clinical Sciences, Malmö University Hospital, Lund University, Malmö, Sweden; ${ }^{2}$ Inserm, U707, UMR-S Inserm and Université Pierre et Marie Curie-Paris6, France;

${ }^{3}$ Department of Health Sciences, Malmö University Hospital, Lund University, Malmö, Sweden

Correspondence to: Dr Basile Chaix, UMR-S 707 Inserm and Université Pierre et Marie Curie-Paris6, 27 rue Chaligny, 75012 Paris, France; chaix@u707.jussieu.fr

Accepted 1 March 2007

\section{ABSTRACT}

Study objective: Previous studies of neighbourhood effects on ischaemic heart disease (IHD) have used census or administrative data to characterise the residential context, most commonly its socioeconomic level. Using the ecometric approach to define neighbourhood social interaction variables that may be relevant to IHD, neighbourhood social cohesion and safety were examined to see how they related to acute myocardial infarction (AMI) mortality, after adjustment for individual and neighbourhood confounders.

Design: To construct social interaction variables, multilevel models were used to aggregate individual perceptions of safety and cohesion at the neighbourhood level. Linking data from the Health Survey in Scania, Sweden, and the Population, Hospital, and Mortality Registers, multilevel survival models were used to investigate determinants of AMI mortality over a three year and nine month period.

Participants: 7791 Individuals aged 45 years and over. Main results: The rate of AMI mortality increased with decreasing neighbourhood safety and cohesion. After adjustment for individual health and socioeconomic variables, low neighbourhood cohesion, and to a lesser extent low safety, were associated with higher AMl mortality. Neighbourhood cohesion effects persisted after adjustment for various neighbourhood confounding factors (income, population density, percentage of residents from low-income countries, residential stability) and distance to the hospital. There was some evidence that neighbourhood cohesion effects on AMI mortality were caused by effects on one-day case-fatality, rather than on incidence. Conclusions: Beyond commonly evoked effects of the physical environment, neighbourhood social interaction patterns may have a decisive influence on $\mathrm{HD}$, with a particularly strong effect on survival after AMI.

Studies have shown that the risk of ischaemic heart disease (IHD) increases with the poverty of the residential neighbourhood, beyond the effects of individual socioeconomic characteristics. ${ }^{1-4}$ The lack of knowledge on the underlying mechanisms is, however, a barrier to the translation of these findings into public health action. ${ }^{5}$

In the literature, hypotheses to explain such neighbourhood effects often refer to the physical environment (availability of healthcare resources and sports facilities; type of food shops and restaurants; built environment characteristics such as walkability and aesthetic quality; air and noise pollution). ${ }^{6}$ In the present study, we emphasise that, beyond the effects of the physical environment, the social-interactional environment ${ }^{7-11}$ may affect IHD risk.

Social-interactional factors refer to the various ways through which residents of a neighbourhood interact with each other, encompassing all aspects of interrelationships such as strong or weaker ties, indifference, aggressive interactions, etc. To consider both stressors and resources, we took into account two neighbourhood social-interactional factors, safety, operationalised as a shared feeling of safety among residents, and social cohesion. Past literature suggests that a feeling of safety is more closely associated with the presence of visible cues of social disorder (litter in the streets, graffiti, groups of young people at street corners, etc. ${ }^{12}$ ) than with the exact victimisation risk. ${ }^{13}$ Accordingly, our indicator may also proxy these neighbourhood circumstances. On the other hand, neighbourhood social cohesion refers to social relationships characterised by extended and interconnected networks of neighbours, a deep and shared feeling of attachment to the neighbourhood, mutually supportive relationships, and capacities to intervene collectively on behalf of the common good. ${ }^{74-18}$

Our hypothesis was that the effects of neighbourhood social interactions on IHD may be mediated by the personal experiences individuals have in their neighbourhood (stressful events, ${ }^{13} 1920$ lack of social support, ${ }^{21} 22$ poor mental wellbeing ${ }^{23}{ }^{24}$, etc.). These experiences ${ }^{25}$ may shape the cognitive and psychological conditions of health behaviour, influencing the odds of health-damaging behaviour, healthcare-seeking habits, and IHD risk.

We have previously shown that a high neighbourhood population turnover (residential instability), corroding networks of neighbours, was associated with an increased IHD risk. ${ }^{26}$ Moreover, people reporting low social support from their neighbours had an increased IHD risk, after adjustment for cardiovascular risk factors and social variables. ${ }^{27}$ Neighbourhood social interactions were, however, directly considered neither in the first study (residential stability is a determinant of social interactions) nor in the second (neighbourhood social support was assessed at the individual level).

Measuring neighbourhood social interactions is challenging. The presence of civil associations, electoral participation, and criminality only constitute indirect proxies of social interactions and/or suffer important biases. ${ }^{13} 152428$ To expand upon previous literature on IHD that assessed neighbourhood social interactions with census or administrative data, ${ }^{28-30}$ we relied on the ecometric approach, ${ }^{31-35}$ taking individuals recruited in a public health survey as raters of their neighbourhood. Examining whether raters from the same neighbourhood agree in their evaluation of the 
residential context, this technique allows one to construct relevant neighbourhood descriptors by integrating individual perceptions into a collective assessment.

Overall, we examined whether neighbourhood social interaction variables such as a shared feeling of safety and cohesion were associated with acute myocardial infarction (AMI) mortality after controlling for individual and neighbourhood sociodemographic characteristics. Second, we assessed whether neighbourhood social interaction influences on AMI mortality were caused by effects on incidence or survival after incidence.

\section{METHODS}

\section{Data sources}

The present study was based on the Health Survey in Scania (HSS) conducted in the region of Scania (Sweden) between November 1999 and February 2000. In this postal questionnaire survey, a total of 24922 randomly selected individuals born between 1919 and 1981 received the questionnaire (one-stage sampling frame). Two reminders were sent to the selected individuals to ensure a higher response rate. The response rate was $59 \%$. In the sample, the distributions of age, gender, marital status, education, and healthcare consumption were similar to those of the Scanian population. ${ }^{36}$ Individuals born in countries other than Sweden were, however, underrepresented in the study sample.

A strength of the data was that the following sources from the LOMAS database (Longitudinal Multilevel Analysis in Scania), a large register-based longitudinal database, ${ }^{4}{ }^{26}{ }^{37}$ were linked to the HSS survey at the individual level: (1) the spatial coordinates of the households of individuals on 1 January 2000; (2) information on income of individuals in 1999 from the Population Register; (3) date and causes of death from 1 April 2000 to 31 December 2003 from the National Mortality Register; and (4) diagnoses made at the hospital from 1987 to 2003 from the National Inpatient Register. Moreover, neighbourhood factors determined by aggregating respondents' answers to the HSS survey at the neighbourhood level, and neighbourhood factors computed from an extraction of the LOMAS database, were merged to the HSS sample (see below).

On the basis of this augmented HSS database, two distinct analytical samples were considered. First, the whole HSS sample with individuals aged 19-81 years in 2000 ( $n=13516$ ) was used when considering individual perceptions of the neighbourhood as the outcome variables. Second, analyses with AMI mortality as the outcome were restricted to HSS participants aged 45 years or over. In this second sample, we excluded 275 individuals who had had an AMI at the hospital over the previous 13 years (code 410 in the International Classification of Diseases (ICD) 9th revision, and code I21 in ICD 10th revision). There were 7791 individuals in this sample.

\section{Measures}

AMI mortality (assessed from the Inpatient and Mortality Registers in LOMAS) was the main outcome variable. It corresponded either to deaths with ICD-10 code I21 for the underlying or contributing causes, or to AMI diagnosed at the hospital (code I21) with a death occurring on the same day. We considered events between 1 April 2000 and 31 December 2003. In a complementary analysis, we took into account the incidence of both fatal and non-fatal AMI (ICD-10 code I21), by combining hospital and mortality data. As the Inpatient and Mortality Registers cover the whole of Sweden, someone having an AMI after moving to another city or region during the follow-up would be identified in the registers.

Using register and survey-based indicators, the individual explanatory covariates considered were age, gender, marital status, diseases previously diagnosed at the hospital, self-rated health, education, income, self-reported financial strain, social participation, perceptions of neighbourhood social interactions (safety and cohesion), smoking, and physical activity.

Age attained in 2000 (from the HSS survey) was divided into 10-year categories (19-29, 30-39, 40-49, 50-59, 60-69, and 70 years or over), but was included as a continuous variable in the analysis of AMI mortality after the linearity of the effect was verified. Marital status (from the HSS survey) was dichotomised in married/cohabiting and living alone.

Regarding previous diseases, separate dummy variables defined from the Inpatient Register in LOMAS according to ICD-9 and ICD-10 indicated whether individuals had received a hospital diagnosis of diabetes, hypertension, and other heart diseases in the 13 years before follow-up. Self-rated health (from the HSS survey) was coded in two classes (poor or fair versus good).

Education (from the HSS survey) was classified in two categories (less than seven years, and seven years and over). Rather than household income, only individual income in 1999 (from the LOMAS database) was available. It was divided into three classes comprising an equal number of individuals. Selfreported financial strain coded in two categories was based on two questions from the HSS survey (difficulties in paying the bills, incapacity to find $€ 1300$ to deal with an unforeseen situation).

Two health behaviours (assessed from the HSS survey) were considered as possible mediators of neighbourhood effects: smoking and physical inactivity. Individuals were categorised as never-smokers, previous smokers, light smokers (less than 10 cigarettes a day), and hard smokers (10 or more cigarettes a day). Regarding physical activity, we distinguished between having no activity during leisure time, and having a moderate physical activity (such as walking or cycling at least four hours a week) or a more intense activity.

Based on a question in the HSS survey, individuals involved in less than four of the following activities over the past year were considered to have a low social participation: ${ }^{38}$ study circle/ course; union meeting; meeting of other organisations; theatre/ cinema; art exhibition; church; sports event; letter to the editor of a newspaper/journal; demonstration; night club/entertainment; large gathering of relatives; and private party. Regarding individual perceptions of neighbourhood social interactions, three-category variables were formed based on questions from the HSS survey asking whether respondents felt safe when going out alone at night in their neighbourhood (really safe; quite safe; not really; or not safe), and whether they felt there was a strong cohesion in their neighbourhood (largely; to a certain extent; not really; or not at all).

Individuals were geocoded to the smallest existing geographical units, corresponding to groupings of parcels (see details in our previous work ${ }^{49}$ ). In the 45-81-year sample, 1533 of those local neighbourhoods were represented. The median number of inhabitants of all ages in those neighbourhoods was 573 (interquartile range 401 to 810).

The whole 19-81-year HSS sample was used to compute our specific neighbourhood social interaction variables. Using the whole sample allowed us to estimate more accurately the levels of cohesion and safety in each neighbourhood. The median number of raters per neighbourhood was six (interquartile range 
Table 1 Descriptive data for the main variables, region of Scania, Sweden, 2000-2003, 45-81-year sample

\begin{tabular}{ll}
\hline AMI deaths (\%) & 0.6 \\
Previous diabetes (\%) & 1.9 \\
Previous hypertension (\%) & 2.7 \\
Previous other heart disease (\%) & 3.7 \\
Poor or fair self-rated health (\%) & 34.8 \\
Males (\%) & 45.6 \\
Age, mean [SD] & $60.5[10.1]$ \\
Living alone (\%) & 24.1 \\
Low education (\%) & 31.0 \\
High economic strain (\%) & 24.4 \\
Perception of low cohesion in the neighbourhood (\%) & $16.4 \%$ \\
Perception of low safety in the neighbourhood (\%) & 13.0
\end{tabular}

AMI, Acute myocardial infarction.

3 to 11 ; minimum 1 ; maximum 92). In $6 \%$ of the neighbourhoods, there was only one rater. Separate multilevel logistic models, i.e. models including a random intercept defined at the neighbourhood level but no other random coefficients, were estimated with feeling really safe and reporting a strong cohesion in the neighbourhood as the outcomes. The neighbourhood random effects of the multilevel models, reflecting neighbourhood levels of perceived safety and cohesion, were used as neighbourhood social interaction variables.

As potential contextual confounders, we first considered neighbourhood income, population density, the percentage of residents from low-income countries, and residential stability. Those factors were computed from an extraction of the LOMAS database including all Scanian inhabitants aged 50-89 years in 1996 (for financial reasons, we did not include precise geocoding information in the whole LOMAS database). Neighbourhood income was defined as mean income of inhabitants, and neighbourhood population density as the number of inhabitants per square kilometre. Following a previously established procedure, ${ }^{40}$ we determined the percentage of residents who were not born in a country with an affluent economy (according to the World Bank classification). Neighbourhood residential stability was measured as the percentage of residents in 1996 who were already living in the same neighbourhood five years before. ${ }^{26}$

Another possible confounding factor was the straight-line distance from each individual's building of residence to the closest of the 10 hospitals of Scania. All neighbourhood variables and the distance variable were divided into four categories.

\section{Statistical analysis}

Considering the 19-81-year sample, multilevel logistic models were estimated with feeling really safe and reporting a strong cohesion in the neighbourhood as the outcomes. Empty multilevel models were first estimated to assess the overall between-neighbourhood variability in perceptions of social interactions. Individual and neighbourhood sociodemographic characteristics were then introduced as explanatory variables. We expressed between-neighbourhood variations ${ }^{41}$ on the odds ratio scale with the interquartile odds ratio, ${ }^{43} 3742$ which quantifies the difference in perceptions between the $25 \%$ of all individuals in neighbourhoods with the lowest odds of positive perceptions and the $25 \%$ of all individuals in neighbourhoods with the highest odds of positive perceptions.

Using the 45-81-year HSS sample, we estimated multilevel random-intercept Weibull survival models to investigate predictors of AMI mortality. The number of participants per neighbourhood (median equal to 4) and the AMI mortality rate (below 1\%) were much too small to allow us to estimate reliably the between-neighbourhood variance in AMI mortality risk. After adjustment for individual variables, neighbourhood safety and cohesion were entered separately into the model, then simultaneously. We successively adjusted the model for the different neighbourhood sociodemographic variables and distance to the hospital. Finally, we assessed whether health behaviour mediated neighbourhood effects on AMI. Using Winbugs 1.4.1, ${ }^{43}$ multilevel models were estimated using a Markov chain Monte Carlo approach.

Then we conducted analyses to assess whether neighbourhood effects on AMI mortality were caused by disparities in incidence or disparities in survival after incidence. CochranArmitage trend tests were applied to the percentage of both fatal and non-fatal incident AMI in the 45-81-year sample and to the rate of one-day case-fatality (AMI for which death occurred on the same day as the AMI) among the 106 AMI cases diagnosed at the hospital to examine whether they were linearly related to the neighbourhood social interaction variables. The number of AMI cases was too small to conduct a multivariable analysis of survival after AMI.

\section{RESULTS}

Descriptive information on the sample of 45-81-year HSS participants is reported in table 1. In our sample, 106 participants out of 7791 had a first-ever incident AMI over the follow-up. Forty-eight participants died from AMI. Sixteen per cent of the participants reported a low cohesion in their neighbourhood. Thirteen per cent of individuals indicated that they did not feel safe at night in their neighbourhood.

In the 19-81-year sample, an empty multilevel logistic model with feeling safe as the outcome indicated that the betweenneighbourhood variance was 0.30 (95\% credible interval (CI) 0.23 to 0.37$)$, yielding an interquartile odds ratio of 3.50 (95\% CI 3.04 to 4.05 ). The corresponding figures were 0.20 (95\% CI 0.14 to 0.26 ) and 2.77 (95\% CI 2.39 to 3.20$)$ for reporting cohesion. There was, therefore, substantial within-neighbourhood agreement in those perceptions of the neighbourhood, supporting the validity of the ecometric approach.

After adjustment for individual characteristics, independent dose-response effects indicated higher odds of feeling safe in affluent neighbourhoods, in neighbourhoods with a low percentage of residents from low-income countries, and in neighbourhoods with a low population density (table 2). After adjustment, reporting neighbourhood cohesion was more common in neighbourhoods with a low rate of people from low-income countries, in low population density areas, and in neighbourhoods with a high residential stability.

Considering a multilevel survival model with AMI mortality as the outcome, a higher risk of mortality was noted for men, older individuals, non-married individuals, people with a low social participation, and low-income individuals (table 3). After adjustment, individual perceptions of safety and cohesion were not associated with AMI mortality.

Whereas the correlation between individual perceptions of safety and cohesion coded in three categories was $0.12(p<0.001)$, the correlation between neighbourhood safety and cohesion among the 1533 neighbourhoods was 0.33 ( $p<0.001$ ). Regarding associations between neighbourhood social interactions and AMI mortality, fig 1 illustrates that the percentage of AMI deaths regularly increased with decreasing neighbourhood cohesion and safety ( $p<0.001$, two-sided Cochran-Armitage trend tests).

Neighbourhood safety and cohesion were entered separately into the model for AMI mortality (table 4). After adjustment for 
Table 2 Individual and neighbourhood effects all adjusted for each other on individual perceptions of neighbourhood social interactions (feeling safe and reporting cohesion in the neighbourhood), as estimated from multilevel logistic models, ${ }^{*}$ region of Scania, Sweden, 2000, 19-81-year sample

\begin{tabular}{|c|c|c|}
\hline & Feeling safe & Reporting cohesion \\
\hline & OR (95\% Cl) & OR $(95 \% \mathrm{Cl})$ \\
\hline Male (versus female) & $3.50(3.22$ to 3.81$)$ & $0.92(0.85$ to 0.99$)$ \\
\hline \multicolumn{3}{|l|}{ Age at baseline (versus 19-29), years } \\
\hline $30-39$ & $1.12(0.97$ to 1.30$)$ & 1.65 (1.42 to 1.92$)$ \\
\hline $40-49$ & 1.14 (0.99 to 1.32$)$ & 2.11 (1.82 to 2.46$)$ \\
\hline $50-59$ & 0.88 (0.76 to 1.02 ) & 2.54 (2.19 to 2.96$)$ \\
\hline $60-69$ & $0.62(0.53$ to 0.73$)$ & $2.77(2.37$ to 3.25$)$ \\
\hline 70 and over & 0.51 (0.43 to 0.61$)$ & $2.69(2.29$ to 3.16$)$ \\
\hline Alone (versus cohabiting) & $1.13(1.03$ to 1.24$)$ & 0.79 (0.73 to 0.87$)$ \\
\hline Low educational attainment (versus high) & 0.62 (0.55 to 0.71$)$ & $1.26(1.13$ to 1.41$)$ \\
\hline High economic strain (versus low) & $0.76(0.70$ to 0.83$)$ & $0.68(0.63$ to 0.74$)$ \\
\hline \multicolumn{3}{|l|}{ Individual income (versus high) } \\
\hline Medium & $0.77(0.70$ to 0.86$)$ & $1.09(0.99$ to 1.19$)$ \\
\hline Low & 0.83 (0.74 to 0.93 ) & $1.08(0.97$ to 1.21$)$ \\
\hline \multicolumn{3}{|l|}{ Neighbourhood income (versus low) } \\
\hline Mid-low & $1.02(0.89$ to 1.16$)$ & $1.10(0.98$ to 1.24$)$ \\
\hline Mid-high & $1.12(0.97$ to 1.28$)$ & $1.09(0.96$ to 1.23$)$ \\
\hline High & $1.38(1.20$ to 1.60$)$ & $1.07(0.94$ to 1.22$)$ \\
\hline \multicolumn{3}{|c|}{ Percentage from low-income countries (versus high) } \\
\hline Mid-high & 1.61 (1.40 to 1.85$)$ & 1.30 (1.15 to 1.47$)$ \\
\hline Mid-low & $1.73(1.50$ to 2.00$)$ & $1.50(1.33$ to 1.70$)$ \\
\hline Low & 1.96 (1.69 to 2.27$)$ & $1.55(1.37$ to 1.76$)$ \\
\hline \multicolumn{3}{|l|}{ Population density (versus high) } \\
\hline Mid-high & 1.24 (1.08 to 1.41$)$ & $1.02(0.90$ to 1.15$)$ \\
\hline Mid-low & $1.60(1.39$ to 1.84$)$ & $1.05(0.93$ to 1.19$)$ \\
\hline Low & 2.45 (2.11 to 2.84 ) & 1.30 (1.14 to 1.48$)$ \\
\hline \multicolumn{3}{|l|}{ Percentage of stable residents (versus low) } \\
\hline Mid-low & $1.06(0.93$ to 1.21$)$ & 1.29 (1.15 to 1.45$)$ \\
\hline Mid-high & $1.05(0.91$ to 1.21$)$ & $1.42(1.27$ to 1.61$)$ \\
\hline High & $1.01(0.87$ to 1.16$)$ & $1.46(1.29$ to 1.65$)$ \\
\hline
\end{tabular}

$\mathrm{Cl}$, Credible interval; OR, odds ratio.

${ }^{*}$ After adjustment for individual and neighbourhood variables, the between-neighbourhood variance was 0.12 ( $95 \% \mathrm{Cl} 0.07$ to 0.17 ) and $0.05(95 \% \mathrm{Cl}<0.01$ to 0.09$)$ for feeling safe and reporting cohesion in the neighbourhood.

individual factors, AMI mortality risk increased with lowering neighbourhood cohesion. AMI risk also tended to be higher in neighbourhoods with a low level of safety, but 95\% CI included the value 1 . As shown in table 5 , only neighbourhood cohesion was associated with AMI mortality when entering neighbourhood safety and cohesion simultaneously into the model. Moreover, neighbourhood cohesion effects on AMI mortality persisted when neighbourhood sociodemographic variables and

Table 3 Individual-level effects* on acute myocardial infarction mortality, as estimated from a multilevel survival model including individual variables only (no neighbourhood variable), region of Scania, Sweden, 2000-2003, 45-81-year sample

\begin{tabular}{ll}
\hline & HR (95\% CI) \\
\hline Previous diabetes & $2.43(0.81$ to 6.15$)$ \\
Previous hypertension & 2.45 (0.97 to 5.66$)$ \\
Previous other heart disease & 1.78 (0.73 to 3.90$)$ \\
Poor or fair self-rated health (versus good) & 1.71 (0.90 to 3.26$)$ \\
Male (versus female) & 4.49 (2.32 to 9.06$)$ \\
Age at baseline (increase of 10 years) & $2.27(1.47$ to 3.12$)$ \\
Alone (versus cohabiting) & $2.44(1.33$ to 4.64$)$ \\
Low educational attainment (versus high) & $0.59(0.29$ to 1.15$)$ \\
High self-reported financial strain (versus low) & 0.74 (0.33 to 1.48$)$ \\
Individual income (versus high) & \\
$\quad$ Medium & 1.89 (0.66 to 5.83$)$ \\
$\quad$ Low & 3.68 (1.38 to 11.63$)$ \\
Low social participation (versus high) & $2.56(1.25$ to 5.85$)$ \\
Perception of low cohesion in neighbourhood (versus medium or high) & 0.78 (0.32 to 1.66$)$ \\
Perception of low safety in neighbourhood (versus medium or high) & 1.52 (0.68 to 3.15$)$
\end{tabular}

\footnotetext{
$\mathrm{Cl}$, Credible interval; $\mathrm{HR}$, hazard ratio.
}

${ }^{*}$ All effects were adjusted for each other. 
Table 4 Effects of neighbourhood social interactions on acute myocardial infarction mortality, as estimated from multilevel survival models adjusted for individual characteristics, region of Scania, Sweden, 2000-2003, 45-81-year sample

\begin{tabular}{|c|c|c|}
\hline & $\begin{array}{l}\text { Explanatory variable: } \\
\text { neighbourhood safety }\end{array}$ & $\begin{array}{l}\text { Explanatory variable: } \\
\text { neighbourhood cohesion }\end{array}$ \\
\hline & HR (95\% Cl) & HR (95\% CI) \\
\hline High & 1.00 & 1.00 \\
\hline Mid-high & $1.90(0.67$ to 5.50$)$ & 1.48 (0.55 to 4.30$)$ \\
\hline Mid-low & 2.07 (0.69 to 6.47 ) & 1.90 (0.73 to 5.54$)$ \\
\hline Low & 2.62 (0.97 to 7.33 ) & 3.43 (1.47 to 9.04$)$ \\
\hline
\end{tabular}

$\mathrm{Cl}$, Credible interval; HR, hazard ratio.

distance to the hospital were introduced, each one into a separate model.

Smoking and physical inactivity were then introduced into the model. Only smoking showed evidence of an association with AMI mortality, but hazard ratios (HR) included the value 1 (HR 1.29; 95\% CI 0.63 to 2.54 for previous smokers, HR 1.43 ; $95 \%$ CI 0.46 to 3.72 for light smokers, and HR $2.28 ; 95 \%$ CI 0.85 to 5.11 for hard smokers versus never-smokers). The inclusion of smoking resulted in a $9 \%$ decrease of the parameter for low versus high neighbourhood cohesion.

Finally, we conducted descriptive analyses to assess whether neighbourhood cohesion effects on AMI mortality were caused by effects on incidence or survival after incidence. In the 45-81-year sample, percentages of both non-fatal and fatal AMI were 1.4\%, $1.1 \%, 2.0 \%$, and $1.0 \%$ in the four quartiles of increasing neighbourhood cohesion; a two-sided Cochran-Armitage trend test $(p=0.69)$
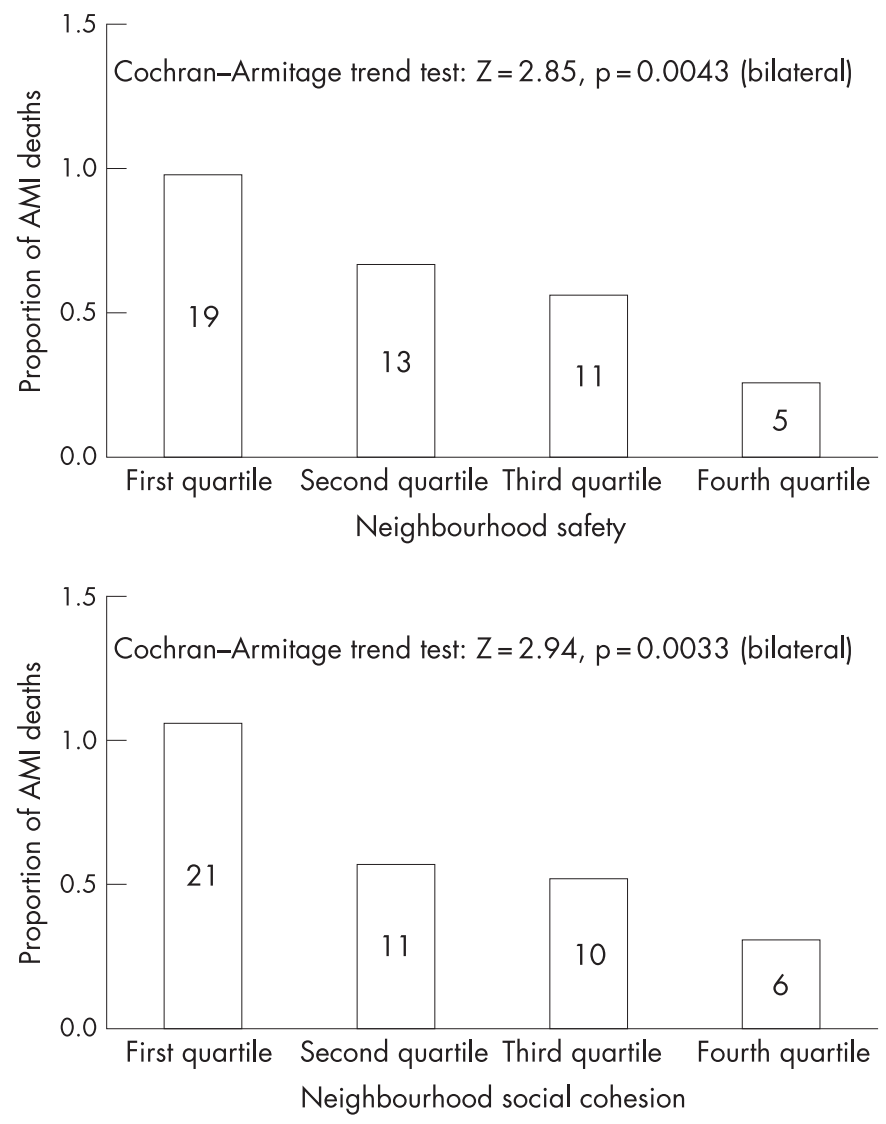

Figure 1 Proportion of deaths by acute myocardial infarction (AMI) in each quartile of neighbourhood safety and neighbourhood cohesion (the number of AMI deaths is reported in each bar), region of Scania, Sweden, 2000-2003, 45-81-year sample. did not identify any trend. Conversely, considering the 106 AMI cases diagnosed at the hospital, percentages of one-day case-fatality were $19.2 \%, 18.5 \%, 7.7 \%$, and $0 \%$ in the four quartiles of increasing neighbourhood cohesion; a two-sided Cochran-Armitage trend test $(p=0.01)$ indicated a significant trend.

\section{DISCUSSION}

The strengths of the present study include the linkage of various data sources at the individual level (survey data, and Population, Hospital, and Mortality Registers) and the use of the ecometric approach to assess neighbourhood social interactions. Following previous debate in the literature, ${ }^{44}{ }^{45}$ we verified that individual and neighbourhood explanatory variables were not too correlated to allow one to disentangle their effects. There were, however, limitations to our study. First, there was a non-response rate of $41 \%$ in the HSS survey. We cannot assert that the estimated association was not distorted at all by a differential nonrepresentativeness of individuals in terms of health between low and high cohesion neighbourhoods. There was, however, no evidence of a major overall non-representativeness as the healthcare consumption of survey participants was similar to that of the whole Scanian population. Second, the short follow-up period of AMI cases resulted in a low statistical power to detect effects on AMI mortality, and limited the investigation of the determinants of one-day case-fatality to descriptive statistics. Third, self-reported data on health behaviour (smoking and physical inactivity) are subject to measurement error. Moreover, we had no biological measurements in our database (cholesterol, hypertension, etc.). Fourth, we could assess the neighbourhood factors used for adjustment only in 1996, i.e. three to four years before the beginning of follow-up.

Whereas previous studies of the effects of neighbourhood social interactions on IHD have relied on census or administrative data, ${ }^{28-30}$ we used the ecometric approach ${ }^{31-35}$ to integrate individual perceptions of neighbourhood functioning into collective assessments of the social-interactional environment. A limitation of our ecometric application is related to the low number of raters available per neighbourhood. This problem may, however, have been mitigated by the strong withinneighbourhood agreement observed in the perception of cohesion. Moreover, the ecometric technique appropriately deals with the uncertainty in defining a contextual variable in neighbourhoods with a low number of raters by shrinking the value of the variable in those neighbourhoods towards the overall variable mean. As an interesting consequence, neighbourhoods classified in the lowest and highest quartiles of neighbourhood cohesion tend to have the highest number of individual raters, and, therefore, the most consistent measures of exposure. Another limitation of our ecometric application is related to the fact that each neighbourhood indicator was only based on a single survey question. ${ }^{32}$ 
Table 5 Neighbourhood cohesion effects on acute myocardial infarction mortality, as estimated from multilevel survival models adjusted for individual characteristics and neighbourhood sociodemographic variables or distance to the hospital (one different model in each column*), region of Scania, Sweden, 2000-2003, 45-81-year sample

\begin{tabular}{|c|c|c|c|c|c|c|}
\hline & $\begin{array}{l}\text { Adjusted for } \\
\text { neighbourhood } \\
\text { safety }\end{array}$ & $\begin{array}{l}\text { Adjusted for } \\
\text { neighbourhood } \\
\text { income }\end{array}$ & $\begin{array}{l}\text { Adjusted for } \\
\text { population } \\
\text { density }\end{array}$ & $\begin{array}{l}\text { Adjusted for } \\
\% \text { from low-income } \\
\text { countries }\end{array}$ & $\begin{array}{l}\text { Adjusted for } \\
\text { residential } \\
\text { stability }\end{array}$ & $\begin{array}{l}\text { Adjusted for } \\
\text { distance to the } \\
\text { hospital }\end{array}$ \\
\hline & HR $(95 \%$ Cl) & HR (95\% CI) & HR $(95 \%$ CI) & HR $(95 \%$ CI) & HR $(95 \%$ CI) & HR $(95 \%$ Cl) \\
\hline \multicolumn{7}{|c|}{ Neighbourhood cohesion } \\
\hline High & 1.00 (ref) & 1.00 (ref) & 1.00 (ref) & 1.00 (ref) & 1.00 (ref) & 1.00 (ref) \\
\hline Mid-high & 1.32 (0.42 to 4.97$)$ & 1.49 (0.52 to 4.55$)$ & 1.43 (0.51 to 3.91 ) & $1.54(0.54$ to 5.15$)$ & 1.31 (0.48 to 3.52 ) & 1.54 (0.61 to 4.78 ) \\
\hline Mid-low & $1.68(0.53$ to 6.26$)$ & $1.89(0.71$ to 6.17$)$ & 1.77 (0.67 to 4.73 ) & $1.95(0.70$ to 6.31$)$ & 1.52 (0.58 to 4.11 ) & 2.05 (0.81 to 6.25 ) \\
\hline High & 1.00 (ref) & 1.00 (ref) & $0.87(0.35$ to 2.16$)$ & 1.05 (0.45 to 2.54$)$ & 1.00 (ref) & 1.52 (0.61 to 3.64 ) \\
\hline Mid-high & 1.99 (0.68 to 8.33 ) & $3.87(0.89$ to 16.95$)$ & 1.26 (0.46 to 3.16 ) & $0.89(0.35$ to 2.25$)$ & 0.77 (029 to 2.20 ) & 1.44 (0.61 to 3.33 ) \\
\hline Mid-low & $2.00(0.65$ to 8.40$)$ & $3.00(0.73$ to 13.20$)$ & 1.68 (0.74 to 4.04$)$ & 1.16 (0.48 to 2.83$)$ & 1.05 (0.43 to 2.92 ) & 1.57 (0.67 to 3.58 ) \\
\hline Low & $2.25(0.80$ to 8.64$)$ & 4.19 (1.13 to 19.34$)$ & 1.00 (ref) & 1.00 (ref) & 1.38 (0.58 to 3.91$)$ & 1.00 (ref) \\
\hline
\end{tabular}

$\mathrm{Cl}$, Credible interval; $\mathrm{HR}$, hazard ratio.

*The neighbourhood cohesion effect was separately adjusted for one neighbourhood sociodemographic variable at a time or distance to the hospital (each column represents a different model).

In our study, strikingly, neither feeling unsafe nor reporting a weak cohesion in the neighbourhood as measured at the individual level was associated with AMI mortality. It was only when we captured the neighbourhood exposure of interest in a more objective way by aggregating at the neighbourhood level individual subjective perceptions of cohesion that an effect emerged. A possible interpretation to investigate in future research is that what matters for AMI mortality is the objective level of cohesion in a neighbourhood, independent of residents' opinions on this issue.

Descriptive analyses suggested that the influence of neighbourhood cohesion resulted from effects on one-day casefatality, rather than on the incidence of first-ever AMI. The latter finding is coherent with our previous study reporting an association between neighbourhood residential stability (as a determinant of cohesion) and survival after AMI. ${ }^{26}$

Possible mechanisms to consider in order to interpret the observed association include: (a) the severity of the AMI; (b) acute triggers of coronary plaque rupture; (c) the time of access to emergency care after AMI; and (d) compliance or not with post-AMI medical recommendations.

Explanation (a) may play a part in our finding. The hypothesis that neighbourhood cohesion effects on AMI mortality are partly caused by the greater severity of AMI in low cohesion neighbourhoods is coherent with the higher oneday case-fatality observed in those areas; however, the medical reasons for this differential severity remain to be understood. Regarding explanation (b), various mechanisms have been suggested as acute triggers of AMI: individual behaviour such as coffee consumption ${ }^{46}$ or heavy physical exertion, ${ }^{47}$ specific psychological states such as anger ${ }^{48}$ or mental stress, $^{49}$ and

\section{What is already known on this subject}

- Studies have shown that the risk of IHD increases with the poverty of the residential neighbourhood, beyond effects of individual socioeconomic characteristics.

- Hypotheses to explain such neighbourhood effects often refer to the physical environment (availability of services for physical activity, food environment, built environment, pollution, etc.). particularly stressful environmental conditions such as in an earthquake. ${ }^{50}$ Therefore, it cannot be excluded that specific triggers or co-triggers of AMI exist in residential environments with inharmonious social interactions (e.g. conflicts with neighbours, incivilities or victimisation, etc.). ${ }^{13}{ }^{19}$ As another speculative hypothesis, residents of low cohesion neighbourhoods may not benefit from a supportive environment that would help them cope with intense worries that could trigger AMI. It is not clear, however, why those mechanisms would result in neighbourhood cohesion effects on one-day casefatality, but not on the overall incidence of first-ever events.

Regarding explanation (c), differences in the time of access to emergency revascularisation procedures ${ }^{51}$ may play a role in neighbourhood cohesion effects on survival after AMI. Neighbourhood cohesion, as a facilitator of the circulation of information between residents, ${ }^{52}$ may increase individuals' recognition of AMI symptoms and awareness of the appropriate action to undertake (immediate call of emergency services). In addition, residents of strongly cohesive neighbourhoods may have more opportunities to receive social support from their neighbours, ${ }^{7122}$ which may be critical to obtain post-AMI emergency care in due time. Finally, explanation (d) also relies on the hypothesis of a greater amount of social support available to the residents of socially cohesive neighbourhoods. Neighbourhood-based social support in its instrumental, informational, appraisal, and emotional forms ${ }^{52}$ may help people adhere to medical recommendations and prescribed treatments.

\section{What this study adds}

- After adjustment for individual health and socioeconomic variables, a low neighbourhood cohesion was associated with a higher AMI mortality; this cohesion effect persisted after adjustment for various neighbourhood confounding factors.

- There was evidence that neighbourhood cohesion effects on AMI mortality were caused by effects on one-day casefatality, rather than on incidence.

- Beyond commonly evoked effects of the physical environment, neighbourhood social interaction patterns may have a decisive influence on IHD, with a particularly strong effect on survival after AMI. 
Explanations (c) and (d) are coherent with the literature that emphasises that social support plays a stronger role in enhancing survival after a cardiac event than in preventing the first incidence of the disease. ${ }^{26} 2753$

Overall, the present study suggests that the neighbourhood social-interactional environment may have a strong impact on IHD.

Acknowledgements: The authors would like to express their gratitude to Statistics Sweden, to the Centre for Epidemiology (National Board of Health and Welfare), and to Region Skåne.

Funding: The present study is part of the LOMAS project ("Socioeconomic disparities in cardiovascular diseases - a longitudinal multilevel analysis"), which is funded by the Swedish Council for Working Life and Social Research (PI JM, Dnr 2003-05809) and the Swedish Research Council (PI JM, Dnr 2004-6155). BC is also supported by the French National Research Agency (Health-Environment program \#00153 05), and by a grant from Région Île-de-France. MR is also supported by an ALF grant from the Swedish Government (Dnr M:B 39923/2005). The present study cannot be attributed to other research funding than those mentioned above.

Competing interests: None.

\section{REFERENCES}

1. Diez Roux AV, Merkin SS, Arnett D, et al. Neighborhood of residence and incidence of coronary heart disease. N Engl J Med 2001:345:99-106.

2. Diez-Roux AV, Nieto FJ, Muntaner $\mathrm{C}$, et al. Neighborhood environments and coronary heart disease: a multilevel analysis. Am J Epidemiol 1997;146:48-63.

3. Sundquist K, Winkleby M, Ahlen $\mathrm{H}$, et al. Neighborhood socioeconomic environment and incidence of coronary heart disease: a follow-up study of 25,319 women and men in Sweden. Am J Epidemiol 2004;159:655-62.

4. Chaix B, Rosvall M, Merlo J. Recent increase of neighborhood socioeconomic effects on ischemic heart disease mortality: a multilevel survival analysis of two large Swedish cohorts. Am J Epidemiol 2007;165:22-6.

5. Schulz AJ, Kannan S, Dvonch JT, et al. Social and physical environments and disparities in risk for cardiovascular disease: the healthy environments partnership conceptual model. Environ Health Perspect 2005:113:1817-25.

6. Diez Roux AV. Residential environments and cardiovascular risk. J Urban Health 2003;80:569-89.

7. Buckner JC. The development of an instrument to measure neighborhood cohesion. Am J Community Psychol 1988;16:771-91.

8. Echeverria SE, Diez Roux AV, Link BG. Reliability of self-reported neighborhood characteristics. J Urban Health 2004;81:682-701.

9. Araya R, Dunstan F, Playle R, et al. Perceptions of social capital and the built environment and mental health. Soc Sci Med 2006;62:3072-83.

10. Poortinga W. Social relations or social capital? Individual and community health effects of bonding social capital. Soc Sci Med 2006;63:255-70.

11. Lochner KA, Kawachi I, Brennan RT, et al. Social capital and neighborhood mortality rates in Chicago. Soc Sci Med 2003;56:1797-805.

12. Geis KJ, Ross CE. A new look at urban alienation: the effect of neighborhood disorder on perceived powerlessness. Soc Psychol 0 1998:61:232-46.

13. Perkins DD, Taylor RB. Ecological assessments of community disorder: their relationship to fear of crime and theoretical implications. Am J Community Psychol 1996;24:63-107.

14. Kasarda JD, Janowitz M. Community attachment in mass society. Am Sociol Rev 1974;39:328-39.

15. Sampson RJ, Byron Groves W. Community structure and crime: testing socialdisorganization theory. Am J Sociol 1989;94:774-802.

16. Sampson RJ, Raudenbush SW, Earls F. Neighborhoods and violent crime: a multilevel study of collective efficacy. Science 1997;277:918-24.

17. Stafford M, Bartley M, Wilkinson R, et al. Measuring the social environment: social cohesion and material deprivation in English and Scottish neighbourhoods. Environ Planning A 2003;35:1459-75.

18. Woldoff RA. The effects of local stressors on neighborhood attachment. Soc Forces 2002;81:87-116.

19. Ewart CK, Suchday S. Discovering how urban poverty and violence affect health: development and validation of a Neighborhood Stress Index. Health Psychol 2002;21:254-62.

20. Lindstrom M, Merlo J, Östergren PO. Social capital and sense of insecurity in the neighbourhood: a population-based multilevel analysis in Malmö, Sweden. Soc Sci Med 2003:56:1111-20

21. Sampson RJ. Local friendship ties and community attachment in mass society: a multilevel systemic model. Am Sociol Rev 1988:53:766-79.

22. Sampson RJ. What "community" supplies. In: Ferguson RF, Dickens WT, editors.Urban problems and community development. Washington: Brookings Institution Press, 1999:241-92.

23. Chaix B, Merlo J, Subramanian SV, et al. Comparison of a spatial perspective with the multilevel analytic approach in neighborhood studies: the case of mental and behavioral disorders due to psychoactive substance use in Malmö, Sweden, 2001. Am J Epidemiol 2005;162:171-82.
24. Chaix B, Leyland AH, Sabel CE, et al. Spatial clustering of mental disorders and associated characteristics of the neighbourhood context in Malmö, Sweden, in 2001. $J$ Epidemiol Community Health 2006;60:427-35.

25. Aneshensel CS, Sucoff CA. The neighborhood context of adolescent mental health $J$ Health Soc Behav 1996;37:293-310.

26. Chaix B, Rosvall M, Merlo J. Neighborhood socioeconomic deprivation and residential instability: effects on incidence of ischemic heart disease and survival after myocardial infarction. Epidemiology 2007;18:104-11.

27. Chaix B, Isacsson SO, Rastam L, et al. Income change at retirement, neighbourhoodbased social support, and ischaemic heart disease: results from the prospective cohort study "men born in 1914". Soc Sci Med 2007;64:818-29.

28. Sundquist $\mathbf{J}$, Johansson $\mathrm{SE}$, Yang $\mathrm{M}$, et al. Low linking social capital as a predictor of coronary heart disease in Sweden: a cohort study of 2.8 million people. Soc Sci Med 2006:62:954-63.

29. Sundquist $\mathbf{K}$, Theobald $\mathbf{H}$, Yang $\mathbf{M}$, et al. Neighborhood violent crime and unemployment increase the risk of coronary heart disease: a multilevel study in an urban setting. Soc Sci Med 2006;62:2061-71.

30. Stjarne MK, Ponce de Leon A, Hallqvist J. Contextual effects of social fragmentation and material deprivation on risk of myocardial infarction - results from the Stockholm Heart Epidemiology Program (SHEEP). Int J Epidemiol 2004;33:732-41.

31. Raudenbush SW. The quantitative assessment of neighborhood socia environments. In: Kawachi I, Berkman LF, editors.Neighborhoods and health. New York, NY: Oxford University Press, 2003:112-31.

32. Raudenbush SW, Sampson RJ. Ecometrics: toward a science of assessing ecological settings, with application to the systematic social observation of neighborhoods. Sociol Methodol 1999:29:1-41.

33. Gauvin L, Richard L, Craig CL, et al. From walkability to active living potential: an "ecometric" validation study. Am J Prev Med 2005;28:126-33.

34. Caughy M0, O'Campo PJ, Patterson J. A brief observational measure for urban neighborhoods. Health Place 2001;7:225-36.

35. Craig CL, Brownson RC, Cragg SE, et al. Exploring the effect of the environment on physical activity: a study examining walking to work. Am J Prev Med 2002;23:36-43.

36. Carlsson F, Merlo J, Lindstrom M, et al. Representativity of a postal public health questionnaire survey in Sweden, with special reference to ethnic differences in participation. Scand J Public Health 2006;34:132-9.

37. Chaix B, Rosvall M, Lynch J, et al. Disentangling contextual effects on cause-specific mortality in a longitudinal 23-year follow up study: impact of population density or socioeconomic environment? Int J Epidemiol 2006;35:633-43.

38. Ali SM, Merlo J, Rosvall M, et al. Social capital, the miniaturisation of community, traditionalism and first time acute myocardial infarction: a prospective cohort study in southern Sweden. Soc Sci Med 2006;63:2204-17.

39. Chaix B, Rosvall M, Merlo J. Assessment of the magnitude of geographic variations and socioeconomic contextual effects on ischaemic heart disease mortality: a multilevel survival analysis of a large Swedish cohort. J Epidemiol Community Health 2007;61:349-55.

40. Beckman A, Hakansson A, Rastam L, et al. The role country of birth plays in receiving disability pensions in relation to patterns of health care utilisation and socioeconomic differences: a multilevel analysis of Malmo, Sweden. BMC Public Health 2006;6:71.

41. Merlo J. Multilevel analytical approaches in social epidemiology: measures of health variation compared with traditional measures of association. J Epidemiol Community Health 2003;57:550-2.

42. Merlo J, Chaix B, Ohlsson $\mathrm{H}$, et al. A brief conceptual tutorial of multilevel analysis in social epidemiology - using measures of clustering in multilevel logistic regression to investigate contextual phenomena. J Epidemiol Community Health 2006;60:290-7.

43. Smith AFM, Roberts GO. Bayesian computation via the Gibbs sampler and related Markov chain Monte Carlo methods. J R Stat Soc Ser B Stat Methodol 1993:55:3-23.

44. Oakes JM. Commentary: advancing neighbourhood-effects research - selection, inferential support, and structural confounding. Int J Epidemiol 2006;35:643-7.

45. Merlo J, Chaix B. Neighbourhood effects and the real world beyond randomized community trials: a reply to Michael J. Oakes. Int J Epidemiol 2006;35:1361-3.

46. Baylin A, Hernandez-Diaz S, Kabagambe EK, et al. Transient exposure to coffee as a trigger of a first nonfatal myocardial infarction. Epidemiology 2006;17:506-11.

47. Hallqvist $\mathbf{J}$, Moller $\mathbf{J}$, Ahlbom A, et al. Does heavy physical exertion trigger myocardial infarction? A case-crossover analysis nested in a population-based casereferent study. Am J Epidemiol 2000;151:459-67.

48. Moller J, Hallqvist J, Diderichsen $\mathrm{F}$, et al. Do episodes of anger trigger myocardial infarction? A case-crossover analysis in the Stockholm Heart Epidemiology Program (SHEEP). Psychosom Med 1999;61:842-9.

49. Strike PC, Steptoe A. Behavioral and emotional triggers of acute coronary syndromes: a systematic review and critique. Psychosom Med 2005;67:179-86.

50. Suzuki S, Sakamoto S, Koide M, et al. Hanshin-Awaji earthquake as a trigger for acute myocardial infarction. Am Heart J 1997;134:974-7.

51. McGinn AP, Rosamond WD, Goff DC Jr, et al. Trends in prehospital delay time and use of emergency medical services for acute myocardial infarction: experience in 4 US communities from 1987-2000. Am Heart J 2005;150:392-400.

52. Berkman LF, Glass T. Social integration, social networks, social support, and health. In: Berkman LF, Kawachi I, editors.Social epidemiology. Oxford, UK: Oxford University Press, 2000:137-73.

53. Vogt TM, Mullooly JP, Ernst D, et al. Social networks as predictors of ischemic heart disease, cancer, stroke and hypertension: incidence, survival and mortality. J Clin Epidemiol 1992;45:659-66. 
development in epidemiology, community medicine or public health.

This book proposes a scheme for the critical appraisal of epidemiological studies and trials. This approach starts by describing the methods and results. Then, it considers three possible non-causal explanations: "observation" bias, confounding and chance. This system forces the investigator or the reader to think about the quality of the data and design and to appraise critically whether a truly causal explanation can be accepted. The aim is to build a logical system of critical appraisal, to allow readers to evaluate studies and to carry out their own studies more effectively. This book emphasises the central importance of causation and unifies the often different approaches used in epidemiology, clinical trials, and evidence-based medicine.

After introducing the concept of causation (chapter 1), the author moves to the types of design used to support a causal relationship (chapter 2) and to issues in the presentation of results that are relevant for causal inference (chapter 3). The central role of measures for attributable proportions and attributable benefits are among the great strengths of this chapter. Chapter 4 deals with the selection of subjects. Then the author devotes three chapters to potential non-causal explanations of findings: observation bias (chapter 5), confounding (chapter 6), and random error (chapter 7). Chapter 8 introduces meta-analysis. Chapter 9, "the diagnosis of causation", addresses the issues of internal and external validity providing a comprehensive 20 -item check-list to appraise the validity of a study systematically. The second section of this chapter reviews the hierarchy of designs and criticises some usual concepts of evidence-based medicine. The six final chapters are centred each on an example of how to appraise a study critically. In these chapters, sections of the original papers are reproduced, the 20-item check-list is thoroughly applied to each example and, very interestingly, a summary of further developments on the topic after the publication of the selected paper is provided.

The style of the book deserves praise for its clarity, applicability, and interest. Excellent examples have been selected representing a very good source for teaching material. The book includes self-test questions (five to 12 questions at the end of each chapter), with the solutions explained at the end. Statistical methods are presented clearly without complex mathematics. More detailed explanations and worked examples are summarised in the appendix.

A major limitation is the omission of recent developments in confounding (ie counterfactuals, causal graphs, colliders). One of the worked examples (obesity confounding the association between exercise and myocardial infarction) missed the fact that obesity may also be an intermediate step in the causal chain. Only later, after more than 40 pages about confounding (page 204), the author explains in another context that a factor in the causal pathway is not a confounder. The author acknowledges that "in much current literature, the main result will come from (...) a multivariate analysis" (page 326), but multivariate methods are dealt with very briefly. The concept of hazard ratio is practically absent. In contrast, some recent books have been successful in handling multivariate methods in a friendly manner (Katz, Multivariate analysis, 2nd ed, Cambridge University Press). After many pages devoted to trials, no mention of equivalence (non-inferiority) trials is made, which would have been very useful for some of the examples presented. The author uses the term "observation bias" instead of "information bias". Unifying terminology in epidemiology is important. The Dictionary of Epidemiology admits observational bias, but not "observation bias". "Information bias" would perhaps have been better.

Miguel Angel Martinez-Gonzalez

\section{CORRECTIONS}

doi:10.1136/jech.2006.056960corr1

The order of the authors of the paper by Chaix et al in the January issue of JECH were published incorrectly (B Chaix, M Lindström, J Merlo, and M Rosvall. Neighbourhood social interactions and risk of acute myocardial infarction. J Epidemiol Community Health 2008;62:62-8). J Merlo is in fact the last author of the paper. The publisher apologises for this mistake.

doi:10.1136/jech.2006.056341 corr1

In the December issue of JECH there were some errors in the published paper by Cohen et al (Cohen J, Bilsen J, Fischer S, et al. End-of-life decision-making in Belgium, Denmark, Sweden and Switzerland: does place of death make a difference? J Epidemiol Community Health 2007;61:1062-8). A corrected version of the manuscript has been posted online as a data supplement to the online article. This can be found at: http:// jech.bmj.com/cgi/data/61/12/1062/DC1/1. 